\title{
结合密集连接块和自注意力机制的腺体细胞分割方法
}

\author{
赵宝奇 ${ }^{1)}$ ，尉飞(1)，孙军梅 ${ }^{1,2)^{*}}$ ，李秀梅 ${ }^{1)}$, 袁珑 ${ }^{1)}$, 肖蕾 ${ }^{2)}$ \\ ${ }^{1)}$ (杭州师范大学信息科学与工程学院 杭州 311121) \\ ${ }^{2)}$ (福建省软件评测工程技术研究中心 厦门 361024) \\ (junmeisun@hznu.edu.cn)
}

\begin{abstract}
摘 要：针对目前常用的细胞分割方法在对腺体细胞进行分割时易出现误分割和分割不精细的问题，提出一种以 U-Net 为基本框架, 结合密集连接块和自注意力机制的腺体细胞分割模型. 首先将 U-Net 结构中卷积层组合构建成密 集连接块，以不同尺度从图像中提取信息; 然后在解码端引人自注意力机制，通过对局部特征建立丰富的上下文依 赖模型, 抑制不必要的特征传播, 提高腺体细胞分割的精度. 在 2015 MICCAI 腺体分割挑战赛数据集上的实验结果 表明, 与 U-Net 等其他模型相比, 在增加少量参数的情况下, 该模型在 $F_{1}$ 值、Mean Dice 和 Hausdorff 距离评价指标 上均具有较大的提升.
\end{abstract}

关键词：腺体细胞分割; 密集连接块; 语义分割; U-Net; 自注意力机制

中图法分类号: TP391.41 DOI: 10.3724/SP.J.1089.2021.18625

\section{Glandular Cell Segmentation Method Combined with Dense Connective Blocks and Self-Attention Mechanism}

\author{
Zhao Baoqi ${ }^{1)}$, Yu Fei ${ }^{1)}$, Sun Junmei ${ }^{1,2)^{*}}$, Li Xiumei ${ }^{1)}$, Yuan Long ${ }^{1)}$, and Xiao Lei ${ }^{2)}$ \\ 1) (School of Information Science and Engineering, Hangzhou Normal University, Hangzhou 311121) \\ 2) (Engineering Research Center for Software Testing and Evaluation of Fujian Province, Xiamen 361024)
}

\begin{abstract}
Currently used cell segmentation methods are easily to cause the problem of missegmentation and impreciseness for glandular cell segmentation. A glandular cell segmentation model based on U-Net network is proposed which combines dense connective blocks and self-attention mechanism. Firstly, the convolution layers in the U-Net structure are combined to form the dense connective blocks, so that the information can be extracted from the image at different scales. Then the self-attention mechanism is introduced at the decoder to establish a rich context-dependent model for local features to suppress unnecessary feature propagation and improve the accuracy of glandular cell segmentation. The experimental results on the 2015 MICCAI Gland Segmentation Challenge dataset show that the proposed model, with a small number of extra parameters, can achieve improved performance in terms of $F_{1}$-score, Mean Dice coefficient, and Hausdorff distance compared with other U-Net based methods.
\end{abstract}

Key words: glandular cell segmentation; dense connective blocks; semantic segmentation; U-Net; self-attention mechanism

收稿日期: 2020-09-01; 修回日期: 2021-02-06. 基金项目：国家自然科学基金(61571174); 福建省软件评测工程技术中心开放课 题(ST2019004); 杭州市科技计划(20201203B124). 赵宝奇(1992-), 男, 硕士研究生, 主要研究方向为医学图像处理、深度学习; 尉飞 (1995-), 男, 硕士研究生, 主要研究方向为医学图像处理、深度学习、机器学习; 孙军梅(1974一), 女, 博士, 副教授, 硕士生导师, CCF 会员, 论文通讯作者, 主要研究方向为智能医学信息处理、智能软件工程; 李秀梅 (1978一), 女, 博士, 教授, 硕士生导师, 主要 研究方向为时频分析及应用、压缩感知、机器学习; 袁珑(1997一), 男, 硕士研究生, 主要研究方向为对抗样本、深度学习; 肖蕾 (1979-), 女, 博士, 副教授, 主要研究方向为医学图像处理、智能软件工程. 
近年来，腺癌成为严重损害人类健康的恶性 肿瘤之一. 分析腺体图像结构的大小、形状和其他 一些形态表现, 可以作为腺癌病情诊断的依据 ${ }^{[1]}$, 因此高效、准确地分割腺体细胞图像成为临床的迫 切要求. 以往都是由专业病理学专家对腺体图像 进行评估，但人工评估存在主观性强、工作量大和 费时等问题. 近几年，通过计算机定量和客观分析 组织病理学图像的计算方法得以发展 ${ }^{[2-3]}$. 其中, 腺体图像语义分割, 即机器从腺体图像中对腺体 细胞进行识别并自动分割, 成为进行腺体图像分 析的基础. 临床实践中利用合适的腺体细胞自动 分割方法, 可以提高分割效率和可靠性，减少病理 学家的工作量.

早期的计算机细胞分割方法大多基于阈值和 形态学运算 ${ }^{[4]}$, 这些方法在复杂的背景下难以找到 可靠的阈值. 而近几年基于深度学习的医学图像 分割方法由于其良好的分割性能, 已成为医学图 像分割的基准方法 ${ }^{[5-6]}$, 其中最经典的方法是编解 码器 (U-Net $)^{[5]}$, U-Net 编码器遵循卷积网络的典型 结构, 从图像中提取空间特征, 解码器根据编码器 特征构建分割图. 目前常用的细胞核和腺体细胞 图像分割方法 ${ }^{[7-9]}$ 主要是采用 U-Net 或其变体, 利 用编码器提取特征, 从低分辨率特征图中恢复高 分辨率预测。

然而，目前自动分割腺体细胞仍然具有挑战 性：(1) 存在大量的细胞遮挡和簇，很容易造成分 割过度和分割不足; (2) 边界模糊和染色不一致, 使图像不可避免地包含无法区分的腺体细胞，可 能引人主观标注和错误标注, 难以获得稳健和客 观的分割结果 ${ }^{[10]}$; (3) 细胞形态、大小和密度在不 同细胞类型和器官之间存在变化, 需要分割方法 具有良好的泛化能力. 但 U-Net 网络在下采样过程 中会降低分割任务中边界的定位精度, 在上采样 过程中没有考虑像素之间的关系. 此外，当物体具 有不同的颜色、纹理和形状时，尤其在腺体细胞图 像中，无法取得令人满意的分割效果.

为了解决现有腺体细胞自动分割方法中存在 的问题，本文提出一种基于密集连接块和自注意 力机制的腺体细胞分割方法, 改进和优化了 U-Net 结构. 改进后的网络通过在卷积层组合构建密集 连接块, 在不改变既有网络层数的情况下, 能够从 不同尺度学习细胞特征, 最大限度地提取图像特 征, 解决了腺体细胞大小不一以及形态和密度不 同所造成的分割精度不高的问题. 此外，改进后的 网络在解码端引入了自注意力机制, 能够对局部
特征建立丰富的上下文依赖模型，从而对远程依 赖关系进行建模，抑制不必要的特征传播，提高细 胞的精确定位; 进而提高腺体细胞分割的精度, 解 决现有腺体细胞自动分割网络中没有充分利用全 局视野中细胞和细胞之间的关系、细胞的定位精度 不高, 且卷积运算导致局部感受野, 从而导致出现 类内不一致性、影响识别准确性的问题.

本文的主要工作如下.

（1）提出在卷积层组合构建密集连接块, 从不 同尺度获取目标特征，以提高腺体细胞分割的准 确性.

（2）在网络解码端引人自注意力模块，通过对 局部特征建立丰富的上下文依赖模型，对远程依 赖关系进行建模，以抑制不必要的传播，提高腺体 细胞分割的特征表示.

(3) 组合密集连接块与自注意力机制以改进 U-Net 模型, 在标准数据集上取得了较好结果, 且 参数较少.

\section{1 相关工作}

\section{1 医学图像分割}

目前, U-Net ${ }^{[5]}$ 、全卷积神经网络(fully convolutional networks, $\mathrm{FCN})^{[11]}$ 和 DeepLab ${ }^{[12]}$ 都是较先 进的图像分割模型. 由于在 U-Net 的编解码器体系 结构中训练网络所需的训练样本数量、内存和计算 时间方面都优于早期的分割方法, 因此很多医学 图像分割方法都是基于 U-Net的变体, 致力于提高 医学图像分割精度和效率. 如 H-DenseUNet ${ }^{[13]}$ 结 合了密集连接网络和 U-Net 的思想，用于 3D 肝脏 和肿瘤分割. UNet $++^{[14]}$ 通过在不同层之间使用密 集的跳跃连接连接编码器和解码器. FU-Net ${ }^{[15]}$ 提 出了一种动态加权的交叉熵损失来改进 U-Net. ANU-Net ${ }^{[16]}$ 重新设计了嵌套的 U-Net结构，集成不 同级别的特征, 以获得更高的分割性能.

\section{2 腺体细胞分割}

在细胞分割中，文献[17]提出了将复杂的多通 道区域和边界与侧面监督相结合的框架, 第 1 次实 现了细胞实例分割. 文献[9]对这项工作进行了扩 展，通过加人附加的边界信息以提高分割性能. MIMONet ${ }^{[18]}$ 通过尺寸裁剪将原图加人每个下采样 模块, 实现原始信息的多层输人, 并通过反卷积每 个上采样的特征恢复到原图大小，再经过通道合 并输出, 实现了特征的多层输出. MDU-Net ${ }^{[19]}$ 提出 在 U-Net 中同时增加 3 个多尺度密集连接，即密集 
编码器、密集编译器以及它们之间的连接, 以提升 细胞的分割精度.

\section{3 注意力模块}

注意力模块可以对远程依赖关系进行建模, 广泛应用于计算机视觉等相关领域 ${ }^{[20-21]}$. 文献[22] 首次提出了提取输人的全局依赖关系的自注意力 机制, 并将其用于机器翻译. 在文献[23]中提出的 SE(squeeze-and-excitation)注意力机制显式地建模 特征通道之间的相互依赖关系，通过学习的方式 自动获取每个特征通道的重要程度，然后依照该 重要程度提升有用特征，并抑制对当前任务用处 较小的特征. 在 SE 注意力机制的基础上，文献[24] 提出了 $\operatorname{scSE}$ (spatial-channel sequeeze \& excitation) 注意力机制, 提出 2 种方法的变体, 其中一种是通 过压缩空间信息获得衡量通道重要性的指标, 并 对原始特征进行重标定; 另一种是通过压缩通道 信息获得衡量空间位置重要性的指标，并对原始 特征进行重标定; 最后同时进行上述 2 种不同重标 定操作, 并将它们的结果按照某种策略进行结合. 由于大尺寸输人特征会保留相关的空间信息，而 SE 中的全局池化操作可能无法正确地捕获这些 空间信息，文献[25]提出了通过从 3 个不同维度 进行池化操作，学习跨不同通道映射之间的相互 依赖性，并将上下文空间和通道进行结合重新标 定输人特征. 文献[26]提出的 DANet(dual attention networks)注意力机制, 通过自注意力机制捕获上下 文信息依赖，自适应地整合局部特征和全局信息.

不同于以往的工作，针对腺体细胞图像在不 同类型和器官之间细胞形态、大小和密度存在变 化, 以及染色不一致等特点, 本文提出了将卷积操 作构建为密集连接块，在不改变原有网络层数的 情况下, 最大限度地提取细胞图像的特征, 并将自 注意力机制首次应用在腺体细胞分割任务中，以 捕捉丰富的上下文信息，提高细胞分割的精度.

\section{2 网络模型的构建}

如图 1 所示, 在腺体细胞图像中, 有很多不规 则且大小不一的细胞，并存在染色不一致的问题. 因此，为了提高腺体细胞分割的准确性，本文以 U-Net 为基本框架, 对网络模型进行了改进和优 化. 通过扩展 U-Net 的卷积层构建密集连接块，以 解决规则不一的细胞分割问题. 在解码器端, 引入 位置注意力模块和通道注意力模块, 自适应聚合 上下文信息，提高细胞分割的特征表示.
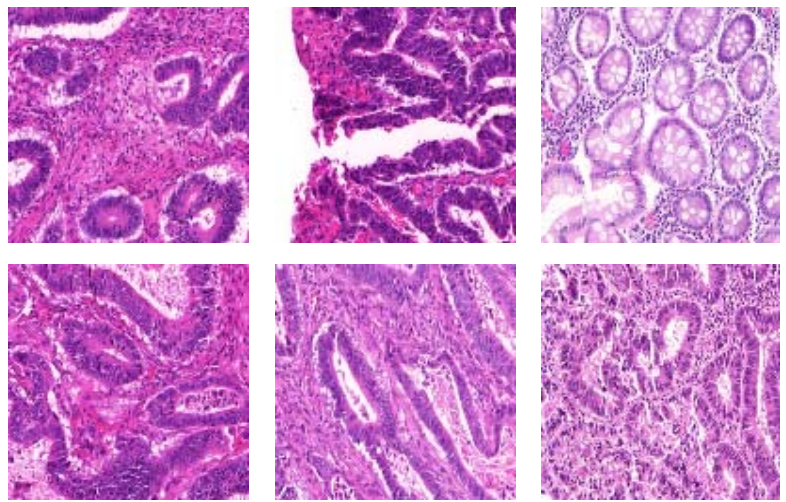

图 1 腺体细胞图像

\section{1 网络结构}

改进后的网络结构如图 2 所示. 在编码器端, 输人腺体细胞图像经过密集连接块, 再经过 $2 \times 2$ 的最大池化, 池化后的输出作为下一次的输人, 如 此 4 次后的输出结果输人第 5 个密集连接块, 再经 过一个 $3 \times 3$ 的反卷积后的输出作为解码端密集连 接块的输人; 在解码器端, 把经过密集连接块生成 的特征图送人注意力模块生成新的特征图, 且和 编码器端经跳跃连接生成的特征图进行相加操作, 然后经过 $3 \times 3$ 的反卷积, 再送人密集连接块, 如此 再循环 4 次, 最后将网络生成的高分辨率特征图通 过 $1 \times 1$ 的卷积映射转化为通道数为 2 的特征图, 送 人 Softmax 分类器以获得每个像素为细胞的概率 值, 得到细胞的分割概率图.

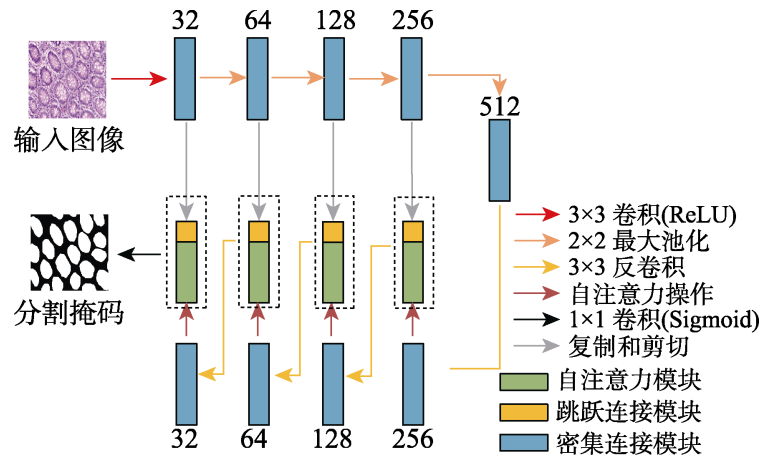

图 2 基于密集连接块和自注意力机制的改进 U-Net 模型

\section{2 密集连接块}

获取多尺度信息对于医学图像分割是至关重 要的. 本文在卷积层组合构建密集连接块, 利用不 同核大小的卷积层来获得图像中不同尺度的兴趣 点，满足规则不一的细胞分割问题.

在 U-Net 架构中，在每个池化层和反卷积层， 使用 2 个串联的 $3 \times 3$ 卷积层. 这 2 个串联的 $3 \times 3$ 卷积运算类似于一个 $5 \times 5$ 卷积运算，在获得尺度 信息上比较单一. 为了保留更多的不同尺度信息, 
本文构建了密集连接块, 密集连接块结构如图 3 所 示. 用密集连接块代替卷积层, 每个 $3 \times 3$ 卷积组成 一层, 将每经过一次卷积的特征图保留下来, 并对 每层的输出再进行密集连接, 然后做残差连接, 最 后合并残差连接、密集连接块所有输出的特征通 道. 这样的构建方法使得有 2 路去捕获信息, 一路 使用一个 $3 \times 3$ 卷积获得小尺度目标; 另一路采用 2 个 $3 \times 3$ 卷积, 即一个 $5 \times 5$ 卷积获得大尺度目标. 改 进后的网络模型可以在不同尺度上获取图像信息, 并可以去理解一些额外的空间信息.

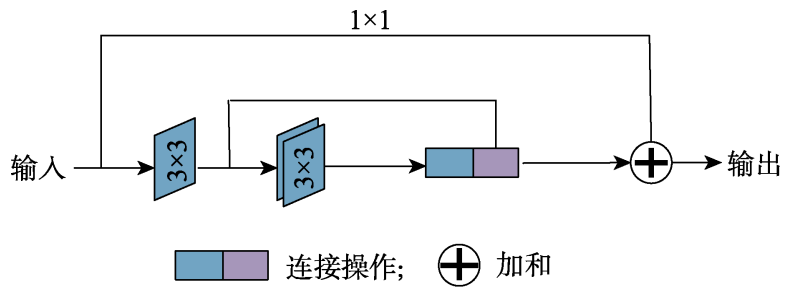

图 3 密集连接块

\section{3 自注意力机制}

卷积运算会导致局部感受野，因此对应于具 有相同标签的像素特征可能具有一些差异, 这些 差异导致了类内不一致性，影响了识别的准确性. 为了解决这个问题, 本文在网络的解码器端引入 自注意力机制，建立具有注意机制的特征关联，以 获取全局上下文信息. 如图 4 所示，包括位置注意 力模块和通道注意力模块. 解码器中生成的特征 图反馈到位置注意力模块, 位置注意力模块通过 3 个步骤生成新的空间远程上下文信息特征.

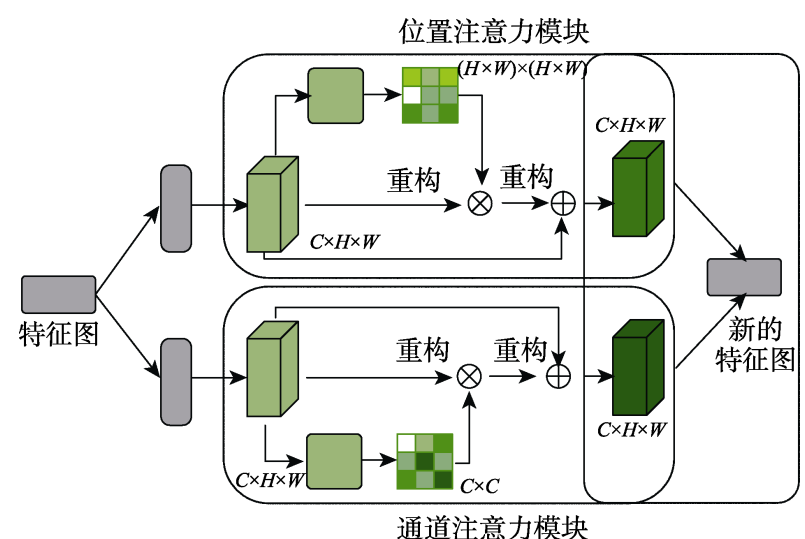

图 4 自注意力模块

Step1. 生成空间注意力矩阵, 该矩阵对特征的任 意 2 个像素之间的空间关系进行建模. 阵乘法

Step2. 在空间注意力矩阵和原始矩阵之间进行矩

Step3. 对上述相乘的结果矩阵和原始特征进行逐
个元素的求和运算, 以获得反映长距离上下文的最终表 示. 同时, 通道注意力模块捕获通道维度中的远程上下 文信息. 获取通道关系的过程和位置注意力模块中获取 位置关系的过程类似，不同之处在于 Step1 是在通道维度 上计算通道注意力矩阵. 最后, 将位置注意力模块和通 道注意力模块进行逐个元素的求和，完成特征融合，以 获得更好的像素级预测的特征表示.

\section{3 .1 位置注意力模块}

如图 5 所示, 对于局部特征图 $\boldsymbol{A} \in \mathbb{R}^{C \times H \times W}$, 先将其送人卷积层生成特征图 $\boldsymbol{B}$ 和 $\boldsymbol{D}$, 其中, $\{\boldsymbol{B}, \boldsymbol{D}\} \in \mathbb{R}^{C \times H \times W}$; 然后将 $\boldsymbol{B}$ 和 $\boldsymbol{D}$ 重构成 $\mathbb{R}^{C \times N}$, 其 中, $N=H \times W$ 为像素点的个数; 最后将 $\boldsymbol{B}^{\mathrm{T}}$ 和 $\boldsymbol{D}$ 矩阵相乘, 再经过 Softmax 层计算出空间特征图 $\boldsymbol{S} \in \mathbb{R}^{C \times N}$. 第 $i$ 个位置在第 $j$ 个位置上的影响为

$$
s_{j i}=\frac{\exp \left(B_{i} \cdot D_{j}\right)}{\sum_{i=1}^{N} \exp \left(B_{i} \cdot D_{j}\right)}
$$

2 个位置的特征表示越相似，则它们之间的相关性 越强.

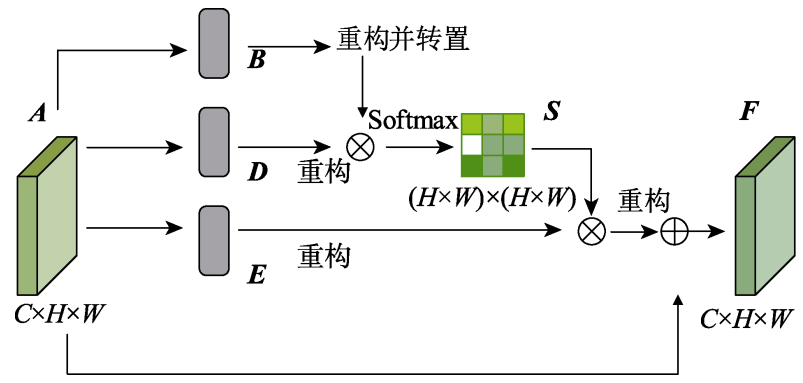

图 5 位置注意力模块

同时，把特征图 $\boldsymbol{A}$ 送到卷积层生成新的特征 图 $\boldsymbol{E}, \boldsymbol{E} \in \mathbb{R}^{C \times H \times W}$, 将特征图 $\boldsymbol{E}$ 重构为 $\mathbb{R}^{C \times N}$; 然 后将 $\boldsymbol{E}$ 和空间特征图 $\boldsymbol{S}$ 的转置矩阵相乘, 并且将 结果重构为 $\mathbb{R}^{C \times H \times W}$, 与特征图 $\boldsymbol{A}$ 做逐个元素求和 的操作

$$
F_{j}=\sum_{i=1}^{N}\left(s_{j i} E_{i}\right)+A_{j}
$$

以获得最终输出 $\boldsymbol{F} \in \mathbb{R}^{C \times H \times W}$. 从式(2)中得出, 在 每个位置处得到的特征图 $\boldsymbol{F}$ 是所有位置上的特征 和原始特征的加权和. 因此, 特征图 $\boldsymbol{F}$ 具有全局 上下文视图，并根据空间特征图选择性地聚合上 下文. 以相似的语义特征实现作为补充，从而提高 了类内紧凑性和语义一致性.

\subsection{2 通道注意力模块}

如图 6 所示，和位置注意力模块不同，通道注 意力模块直接用局部特征图 $\boldsymbol{A} \in \mathbb{R}^{C \times H \times W}$ 计算通道 
特征图 $\boldsymbol{X} \in \mathbb{R}^{C \times C}$. 具体地, 将特征图 $\boldsymbol{A}$ 重构成 $\mathbb{R}^{C \times N}$, 然后对 $\boldsymbol{A}$ 与 $\boldsymbol{A}^{\mathrm{T}}$ 做矩阵相乘, 最后经过 Softmax 层得到通道特征图 $\boldsymbol{X} \in \mathbb{R}^{C \times C}$. 第 $\mathrm{i}$ 个通道 在第 $j$ 个通道上的影响为

$$
x_{j i}=\frac{\exp \left(A_{i} \cdot A_{j}\right)}{\sum_{i=1}^{N} \exp \left(A_{i} \cdot A_{j}\right)}
$$

此外, 对 $\boldsymbol{X}$ 的转置和 $\boldsymbol{A}$ 做矩阵相乘, 将它们 的结构重构成 $\mathbb{R}^{C \times H \times W}$, 然后对特征图 $\boldsymbol{X}$ 和特征 图 $\boldsymbol{A}$ 进行逐个元素求和

$$
F_{j}=\sum_{i=1}^{C}\left(x_{j i} A_{i}\right)+A_{j}
$$

获得最终输出 $\boldsymbol{F} \in \mathbb{R}^{C \times H \times W}$. 式(4)显示每个通道的 最终特征是所有通道的特征和原始特征的加权和, 通过对特征映射之间的长期语义依赖性进行建模， 有助于提高特征的可识别性.

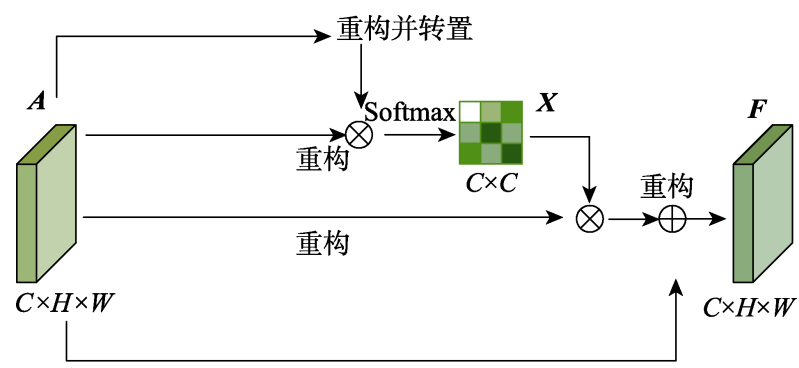

图 6 通道注意力模块

\section{3 .3 注意力模块的特征融合}

为了充分利用远程上下文信息, 本文综合了 位置注意力模块和通道注意力模块的特征. 具体 地，通过卷积层对这 2 个注意力模块的输出进行变 换，并进行逐个元素的求和完成特征融合. 最后将 网络生成的高分辨率特征图通过 $1 \times 1$ 的卷积映射 转化为通道数为 2 的特征图, 送人 Softmax 分类器, 以获得每个像素为细胞的概率值, 进而得到细胞 的分割图。

\section{3 实验及结果分析}

为了验证本文方法是否对腺体细胞分割精度 有较大的提升以及可用性，需要回答以下问题.

问题 1. U-Net 模型的模块为什么选择 2 个 $3 \times 3$ 卷积串联？对最后的分割精度有什么影响？本文提 出的密集连接块应该选择几个 $3 \times 3$ 卷积进行构建?

问题 2. 在 U-Net 模型上引人自注意力机制, 是否会提升分割精度? 组合使用密集连接块和自 注意力机制是否比单一的效果更好?
问题 3. 基于密集连接块和自注意力机制的分 割方法与其他方法相比在分割精度上有无提升? 是否还有其他的优势?

\section{1 数据集}

使用 2015 MICCAI 腺体分割挑战赛数据集 (Glas) ${ }^{[27]}$ 作为实验基准数据集, Glas 数据集由 165 幅 腺体细胞图像组成, 这些图像来自 16 个 H\&E 染色 的大肠癌组织切片. 原始图像大小不一, 而大多数 尺寸为 $775 \times 522$ 像素. 首先将图像尺寸统一调整 为 $512 \times 512$ 像素. 其次将数据集分为训练集和测 试集, 85 幅图像属于训练集, 80 幅图像属于测试集 (测试集 $A$ 包含 60 幅图像, 作为验证集; 测试集 $B$ 包含 20 幅图像, 作为测试集). 训练集中有良性切 片 37 个, 恶性切片 48 个; 测试集 $A$ 中有良性切片 33 个, 恶性切片 27 个; 测试集 $B$ 中有良性切片 4 个, 恶性切片 16 个.

\section{2 评价指标及实验设置}

本文使用与 MICCAI Glas 挑战赛相同的评估 标准对方法的性能进行评估, 包含 $F_{1}$ 值、Mean Dice、目标级别 Hausdorff 距离. 其中, $F_{1}$ 值用来衡 量单个腺体目标的检测精度; Dice 指数用来衡量 2 组样本之间的相似性; Hausdorff 距离用来衡量基 于边界的分割精度.

为确定最佳训练参数, 分别采用不同的批输 人尺寸和初始学习率对 U-Net 原模型进行训练. 实 验结果如图 7 和图 8 所示, 当批输人尺寸为 2 、初 始学习率为 0.0001 时, 模型的精度最高, 因此后 续的实验均选取批输人尺寸为 2 , 初始学习率为 0.0001 .

\section{3 密集连接块实验及结果分析}

为了验证和分析 U-Net 串联使用不同卷积层 个数对模型效果的影响, 分别设置串联的 $3 \times 3$ 卷 积数目为 $1,2,3,4$ 进行实验, 实验结果如表 1 所示. 实验发现, 当串联的 $3 \times 3$ 卷积数目为 2 个时, 分割 精度最好.

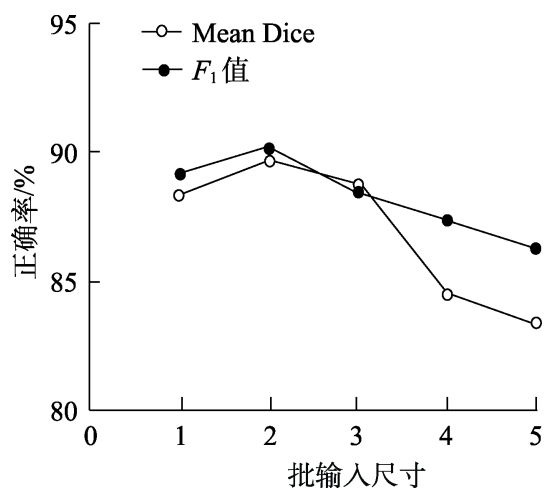

图 7 不同批输人尺寸下训练及验证精度 


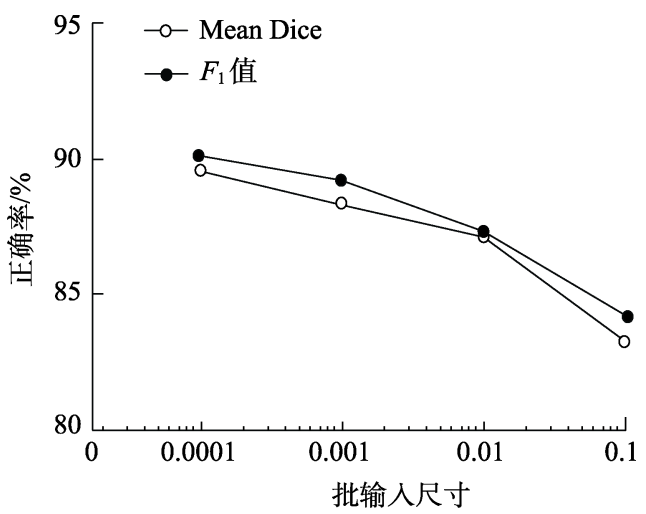

图 8 不同初始学习率下训练及验证精度

表 1 串联不同数目 $3 \times 3$ 卷积的 U-Net 分割效果

\begin{tabular}{cccc}
\hline $3 \times 3$ 卷积数目 & $F_{1}$ 值 & Mean Dice & Hausdorff 距离 \\
\hline 1 & 0.8825 & 0.8432 & 45.52 \\
2 & 0.9011 & 0.8962 & 42.13 \\
3 & 0.8997 & 0.8814 & 43.28 \\
4 & 0.8721 & 0.8278 & 44.64 \\
\hline
\end{tabular}

分析原因, 2 个 $3 \times 3$ 卷积串联等同于 1 个 $5 \times 5$ 卷积, 3 个 $3 \times 3$ 卷积串联等同于 1 个 $7 \times 7$ 卷积, 且 随着网络深度的加深, 感受野越来越大. 所以最开 始的卷积如果太大, 感受野的增大速度将过快, 对 一些小目标无法进行检测. 尤其在细胞图像分割 中, 由于细胞的粘连性, 会把很多细胞簇误认为是 一个大细胞，导致分割精度降低. $F_{1}$ 值反映的是单 个细胞目标的检测精度，能较好地体现把细胞簇 错误分割为单个细胞的情况. 在本实验中，当 $3 \times 3$ 卷积数目为 4 时, $F_{1}$ 值下降比较多. 这是因为当串 联数目为 4 时, 感受野过大, 很容易把很多细胞簇 误认为单个细胞. 而若只用一个 $3 \times 3$ 的卷积, 对于 一些大目标则无法进行完备的检测，且最后的分割 结果边缘非常粗楉, 导致分割精度降低.

通过上述分析可知，单纯靠扩大或减少卷积 的数目并不能达到获取不同尺度特征信息的目的. 对此, 本文在 U-Net 的基础上, 提出构建密集连接 块, 分别用 2 个不同层数的卷积并行获取目标特征 后，将获取的不同特征进行融合，这样可以获取不 同尺度的目标. 为了验证构建的密集连接块对细 胞分割效果的影响，对基于密集连接块的细胞分 割进行了实验. 而且为了更好地与不同层数的卷 积操作分割效果进行对比, 分别设置构建密集连 接块的卷积个数为 $1,2,3,4$. 实验结果如表 2 所示. 从表 1 和表 2 的比较中可以看出, 在相同卷积层数 情况下，使用密集连接块的分割效果更好. 其中,
当卷积层数为 2 时分割精度最高, 较 U-Net 的 $F_{1}$ 值提高 $1.62 \%$, Mean Dice 提高 $1.33 \%$, Hausdorff 缩 短了 1.02. 这也验证了感受野对于分割精度的影 响. 本文提出的密集连接块及后续实验中均使用 2 个串联的 $3 \times 3$ 卷积来构成.

表 2 密集连接块中不同数目 $3 \times 3$ 卷积的分割效果

\begin{tabular}{cccc}
\hline $3 \times 3$ 卷积数目 & $F_{1}$ 值 & Mean Dice & Hausdorff 距离 \\
\hline 1 & 0.8825 & 0.8432 & 45.52 \\
2 & 0.9173 & 0.9095 & 41.11 \\
3 & 0.9032 & 0.8901 & 42.23 \\
4 & 0.8811 & 0.8434 & 43.62 \\
\hline
\end{tabular}

\section{4 引入注意力机制实验及结果分析}

本节依照第 3.2 节确定的超参数, 引人不同的 注意力机制进行训练和交叉验证. 迭代次数为 10000 次.

U-Net 的解码端主要负责上采样的工作，且为 了较好地利用编码器端的信息, 嵌人了跳跃连接, 但对上下文信息没有建立依赖关系. 为了解决现 有腺体细胞自动分割网络中没有充分利用全局视 野中细胞和细胞之间的关系, 细胞的定位精度不 高等问题, 本文在 U-Net 解码端引人注意力机制. 为了验证和分析不同注意力机制对模型分割效果 的影响, 在 U-Net 解码端分别引入 $\mathrm{SE}^{[23]}, \mathrm{scSE}^{[24]}$, Project\&Excite ${ }^{[25]}$ 和 DANet ${ }^{[26]}$ 等注意力机制进行实 验比较, 实验结果如表 3 所示.

表 3 U-Net 模型上引入不同注意力机制的分割效果

\begin{tabular}{lccc}
\hline \multicolumn{1}{c}{ 注意力机制 } & $F_{1}$ 值 & Mean Dice & Hausdorff 距离 \\
\hline $\mathrm{SE}^{[23]}$ & 0.9024 & 0.8979 & 41.89 \\
$\mathrm{scSE}^{[24]}$ & 0.9123 & 0.8972 & 41.55 \\
Project\&Excite $^{[25]}$ & 0.9154 & 0.9031 & 42.34 \\
DANet $^{[26]}$ & 0.9160 & 0.9043 & 41.05 \\
\hline
\end{tabular}

由实验结果可知，引人不同的注意力机制对 使用 U-Net 进行细胞分割精度均有提升，且使用 DANet 注意力机制时分割精度最高, 说明引人注 意力机制对提升模型分割精度是有帮助的. 因此, 考虑在构成密集连接块的 U-Net 模型上引人注意 力机制方法.

本文在构成密集连接块的 U-Net 模型中同样引 人注意力机制进行实验，以验证利用密集连接块改 进 U-Net 模型后的模型上引人注意力机制是否可以 提升模型的分割效果. 实验结果如表 4 所示. 
表 4 在构成密集连接块的 U-Net 模型上引入 不同注意力机制的分割效果

\begin{tabular}{lccc}
\hline \multicolumn{1}{c}{ 注意力机制 } & $F_{1}$ 值 & Mean Dice & Hausdorff 距离 \\
\hline SE $^{[23]}$ & 0.9188 & 0.9113 & 38.49 \\
scSE $^{[24]}$ & 0.9198 & 0.9095 & 39.62 \\
Project\&Excite $^{[25]}$ & 0.9211 & 0.9112 & 40.12 \\
DANet $^{[26]}$ & 0.9275 & 0.9176 & 37.89 \\
\hline
\end{tabular}

由实验结果可知，在构成密集连接块的 U-Net 模型上引人注意力机制对分割精度有更大的提升, 而且使用 DANet 注意力机制时分割精度也是最高. 以引人同一个注意力机制 DANet 为例, 与只将串 联构建成密集连接块的 U-Net 相比，加人注意力模 块后, $F_{1}$ 值提高 $1.02 \%$, Mean Dice 提高 $0.81 \%$, Hausdorff 距离缩短了 3.22 ; 与只引人 DANet 注意 力机制的 U-Net 模型相比, $F_{1}$ 值提高 $1.15 \%$, Mean Dice 提高 $1.33 \%$, Hausdorff 距离缩短了 3.16 . 即将 串联卷积构建成密集连接块和引人注意力机制组 合使用对模型的分割精度有更大的提升，比单独 将串联卷积构成密集连接块和只引人注意力机制 的分割精度都要更好.

分析原因：(1) 构建的密集连接块可以从不同 尺度获取信息，存在较少的信息丢失问题; (2) DANet 可以自适应地整合局部特征和建立全局依 赖关系. 其中通道注意力模块, 捕获了任意 2 个通 道特征图之间的通道依赖关系，并使用所有通道 特征图的加权和更新每个通道特征图, 最后融合 2 个注意力模块的输出进一步增强特征表示; 位置 注意力模块, 捕获要素图的任意 2 个位置之间的空 间依赖性，具有相似特征的任何 2 个位置可以促进 相互改进，而不关注它们在空间维度上的距离. 基 于以上分析，本文最终采用将 DANet 注意力机制 嵌人到构成密集连接块的 U-Net 模型中，以提升细 胞分割精度.

\section{5 与其他模型分割结果比较及分析}

为了验证本文提出的基于密集连接块和自注 意力机制的腺体细胞分割方法的有效性，本文选 取基础网络 U-Net 模型 ${ }^{[5]}$ 、对 U-Net 拓扑结构提出 改变的 UNet++模型 ${ }^{[14]}$ 、同样使用密集连接思想的 MDU-Net 模型 ${ }^{[19]}$, 以及其他图像语义分割模型 SegNet ${ }^{[28]}$ 在腺体细胞分割精度和参数量上进行定 量比较, 实验结果如表 5 所示. 从定量均值比较结 果可以看出，本文方法在分割精度上取得了更好 的性能, 与 MDU-Net 模型相比, 本文方法的 $F_{1}$ 值 提高 $1.41 \%$, Mean Dice 提高 $1.03 \%$, Hausdorff 距离 缩短了 0.28 ; 与 $\mathrm{UNet}++$ 模型相比, 本文方法的 $F_{1}$
值提高 $1.28 \%$, Mean Dice 提高 $1.52 \%$, Hausdorff 缩 短了 0.79 ; 与 U-Net 模型相比, 本文方法的 $F_{1}$ 值提 高 2.4\%, Mean Dice 提高 2.55\%, Hausdorff 缩短了 2.7; 与 SegNet 模型相比, 本文方法的 $F_{1}$ 值提高 $3.87 \%$, Mean Dice 提高 4.44\%, Hausdorff 缩短了 4.34. 由于本文方法引人了注意力机制, 与 U-Net 模型相比参数量有一定提升, 如表 6 所示, 但与 UNet++和 MDU-Net 模型相比, 参数量较少且分割 精度更高. 实验结果证明, 本文方法对于腺体细胞 分割有比较大的优势.

表 5 不同模型在 Glas 数据集上的分割效果

\begin{tabular}{lclc}
\hline \multicolumn{1}{c}{ 模型 } & $F_{1} \pm$ std & \multicolumn{2}{c}{ Mean Dice \pm std Hausdorff 距离 \pm std } \\
\hline SegNet $^{[28]}$ & $0.888 \pm 0.214$ & $0.8732 \pm 0.186$ & $42.23 \pm 2.24$ \\
U-Net $^{[5]}$ & $0.903 \pm 0.129$ & $0.8921 \pm 0.149$ & $40.59 \pm 1.73$ \\
UNet++ $^{[14]}$ & $0.914 \pm 0.104$ & $0.9024 \pm 0.112$ & $38.68 \pm 1.43$ \\
MDU-Net $^{[19]}$ & $0.913 \pm 0.096$ & $0.9073 \pm 0.089$ & $38.17 \pm 1.32$ \\
本文 & $\mathbf{0 . 9 2 7} \pm \mathbf{0 . 0 8 6}$ & $\mathbf{0 . 9 1 7 6} \pm \mathbf{0 . 0 9 4}$ & $\mathbf{3 7 . 8 9} \pm \mathbf{1 . 2 1}$ \\
\hline
\end{tabular}

注. std 表示标准差, 粗体表示最优结果.

表 6 不同模型的参数量

\begin{tabular}{ll}
\hline \multicolumn{1}{c}{ 模型 } & $10^{-6} \times$ 参数量 \\
\hline SegNet & 12.5 \\
U-Net $^{[5]}$ & $\mathbf{8}$ \\
${\text { UNet }++^{[14]}}^{[2]}$ & $8+1$ \\
MDU-Net & $8+0.015$ \\
本文 & $8+0.003$ \\
\hline
\end{tabular}

注. 粗体表示最优.

图 9 所示为本文方法在测试集上的分割结果. 定性比较也可以看出其对于细胞图像中较小的细 胞分割效果较其他模型有较大的提升, 且边缘分 割效果更好.

\section{4 结 语}

本文针对腺体细胞自身特点以及目前腺体细 胞误分割和分割不精细问题, 提出了一种以 U-Net 为基本框架，结合密集连接块和自注意力机制的 腺体细胞分割方法. 该方法在模型的编码器端构 建密集连接块, 获取不同尺度大小的目标, 解决了 腺体细胞误分割的问题; 在解码端引人自注意力 机制，使之可以自适应地聚合上下文信息，从而提 高细胞分割的特征表示, 对细胞进行更精细地分 割. 实验结果表明, 本文方法比现有方法在腺体细 胞分割精度上有较大的提升, 且对于细胞边缘的 分割更加精细. 但本文方法也存在一定的提升空 间, 如本文方法是根据腺体细胞图像特征在医学 


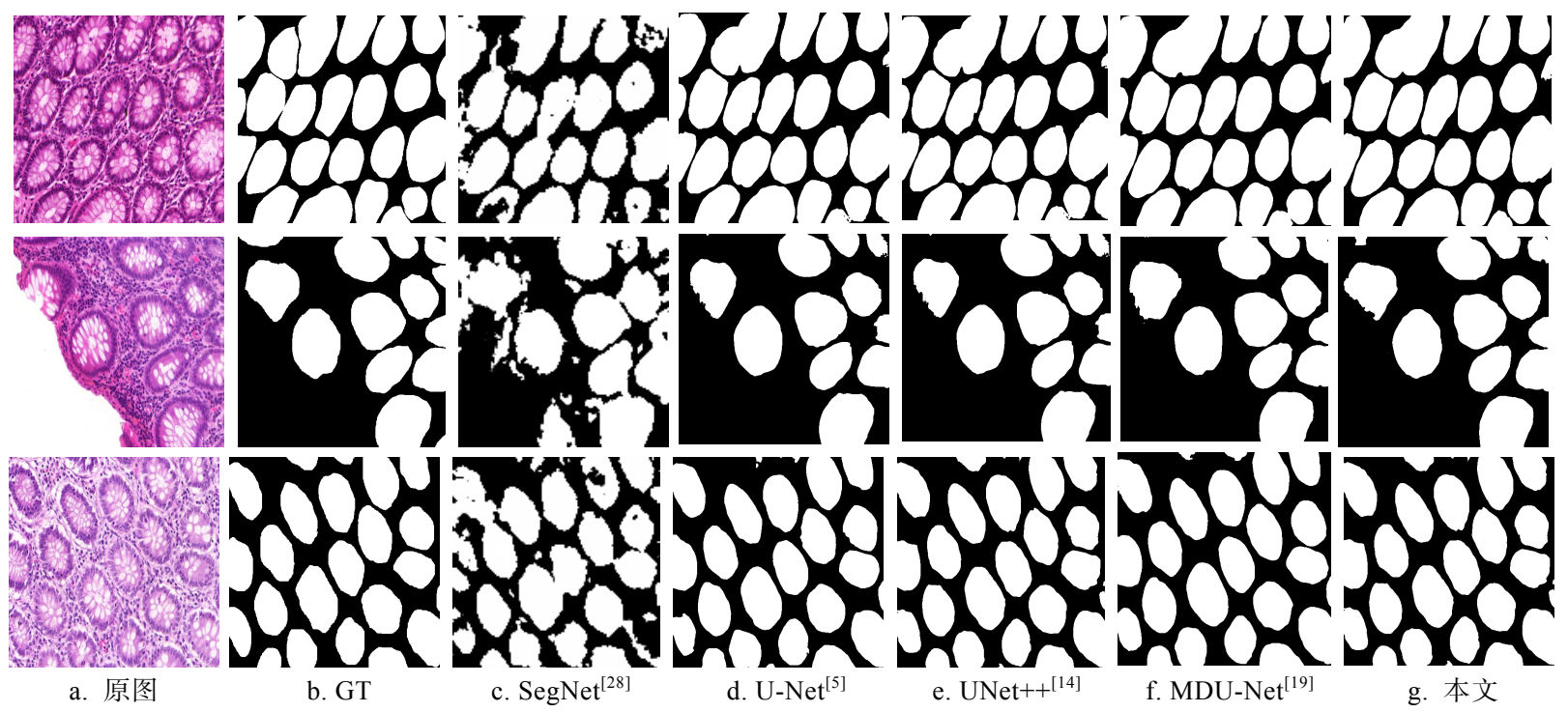

图 9 测试集分割结果实例

图像分割框架 U-Net上所进行的改进和优化，对于 其他图像的分割效果还需通过实验验证. 此外，由 于医学图像标注数据难的局限性, 本文只在 2015 MICCAI 腺体分割挑战赛数据集上进行了实验. 以 后可考虑研究使用无监督学习算法与迁移学习, 利用更多无标签的图像数据来进行网络训练, 进 一步提升网络分割精度.

\section{参考文献(References):}

[1] Gurcan M N, Boucheron L E, Can A, et al. Histopathological image analysis: a review[J]. IEEE Reviews in Biomedical Engineering, 2009, 2: 147-171

[2] Zhang X F, Xing F Y, Su H, et al. High-throughput histopathological image analysis via robust cell segmentation and hashing[J]. Medical Image Analysis, 2015, 26(1): 306-315

[3] Su H, Xing F Y, Kong X F, et al. Robust cell detection and segmentation in histopathological images using sparse reconstruction and stacked denoising autoencoders[C] // Proceedings of the International Conference on Medical Image Computing and Computer-Assisted Intervention. Heidelberg: Springer, 2015: 383-390

[4] Jung C, Kim C. Segmenting clustered nuclei using H-minima transform-based marker extraction and contour parameterization [J]. IEEE Transactions on Biomedical Engineering, 2010, 57 (10): 2600-2604

[5] Ronneberger O, Fischer P, Brox T. U-Net: convolutional networks for biomedical image segmentation[C] //Proceedings of the International Conference on Medical Image Computing and Computer-Assisted Intervention. Heidelberg: Springer, 2015: 234-241

[6] Yi X, Walia E, Babyn P. Generative adversarial network in medical imaging: A review[J]. Medical Image Analysis, 2019, 58: 101552
[7] Graham S, Chen H, Gamper J, et al. MILD-Net: minimal information loss dilated network for gland instance segmentation in colon histology images[J]. Medical Image Analysis, 2019, 52: 199-211

[8] Qu H, Wu P X, Huang Q Y, et al. Weakly supervised deep nuclei segmentation using partial annotation in histopathology images[J]. IEEE Transactions on Medical Imaging, 2020, 39(11): 3655-3666

[9] Xu Y, Li Y, Wang Y P, et al. Gland instance segmentation using deep multichannel neural networks[J]. IEEE Transactions on Biomedical Engineering, 2017, 64(12): 2901-2912

[10] Irshad H, Montaser-Kouhsari L, Waltz G, et al. Crowdsourcing image annotation for nucleus detection and segmentation in computational pathology: evaluating experts, automated methods, and the crowd[C] //Proceedings of the Pacific Symposium on Biocomputing Co-chairs. Lauderdale Lakes: World Scientific, 2015: 294-305

[11] Shelhamer E, Long J, Darrell T. Fully convolutional networks for semantic segmentation[J]. IEEE Transactions on Pattern Analysis and Machine Intelligence, 2017, 39(4): 640-651

[12] Chen L C, Papandreou G, Kokkinos I, et al. DeepLab: semantic image segmentation with deep convolutional nets, atrous convolution, and fully connected CRFs[J]. IEEE Transactions on Pattern Analysis and Machine Intelligence, 2018, 40(4): 834-848

[13] Li X M, Chen H, Qi X J, et al. H-DenseUNet: hybrid densely connected UNet for liver and tumor segmentation from CT olumes[J]. IEEE Transactions on Medical Imaging, 2018, 37(12): 2663-2674

[14] Zhou Z W, Rahman Siddiquee M M, Tajbakhsh N, et al. UNet++: a nested U-Net architecture for medical image segmentation[C] //Proceedings of the Deep Learning in Medical Image Analysis and Multimodal Learning for Clinical Decision Support. Heidelberg: Springer, 2018: 3-11

[15] Jafari M, Li R Z, Xing Y, et al. FU-Net: multi-class image segmentation using feedback weighted U-Net[C] //Proceedings of the International Conference on Image and Graphics. Heidelberg: Springer, 2019: 529-537 
[16] Li C, Tan Y S, Chen W, et al. ANU-Net: attention-based nested U-Net to exploit full resolution features for medical image segmentation[J]. Computers \& Graphics, 2020, 90: 11-20

[17] Xu Y, Li Y, Liu M Y, et al. Gland instance segmentation by deep multichannel side supervision[C] //Proceedings of the International Conference on Medical Image Computing and Computer-Assisted Intervention. Heidelberg: Springer, 2016: 496-504

[18] Raza S E A, Cheung L, Epstein D, et al. MIMONet: gland segmentation using multi-input-multi-output convolutional neural network[C] //Proceedings of the Annual Conference on Medical Image Understanding and Analysis. Heidelberg: Springer, 2017: 698-706

[19] Zhang J W, Jin Y Z, Xu J L, et al. MDU-Net: multi-scale densely connected U-Net for biomedical image segmentation [OL]. [2020-09-01]. https://arxiv.org/pdf/1812.00352.pdf

[20] Shen T, Zhou T Y, Long G D, et al. DiSAN: directional selfattention network for RNN/CNN-free language understanding [C] //Proceedings of the 32nd AAAI Conference on Artificial Intelligence. Palo Alto: AAAI Press, 2018: 5446-5455

[21] Lin G S, Shen C H, van den Hengel A, et al. Efficient piecewise training of deep structured models for semantic segmentation[C] //Proceedings of the IEEE Conference on Computer Vision and Pattern Recognition. Los Alamitos: IEEE Computer Society Press, 2016: 3194-3203

[22] Kendall A, Badrinarayanan V, Cipolla R. Bayesian SegNet: model uncertainty in deep convolutional encoder-decoder ar- chitectures for scene understanding[OL]. [2020-09-01]. https:// arxiv.org/pdf/1511.02680.pdf

[23] $\mathrm{Hu}$ J, Shen L, Sun G. Squeeze-and-excitation networks[C] // Proceedings of the IEEE Conference on Computer Vision and Pattern Recognition. Los Alamitos: IEEE Computer Society Press, 2018: 7132-7141

[24] Roy A G, Navab N, Wachinger C. Concurrent spatial and channel 'squeeze \& excitation' in fully convolutional networks[C] // Proceedings of the International Conference on Medical Image Computing and Computer-Assisted Intervention. Heidelberg: Springer, 2018: 421-429

[25] Rickmann A M, Roy A G, Sarasua I, et al. 'Project \& Excite' modules for segmentation of volumetric medical scans[C] // Proceedings of the International Conference on Medical Image Computing and Computer-Assisted Intervention. Heidelberg: Springer, 2019: 39-47

[26] Fu J, Liu J, Tian H J, et al. Dual attention network for scene segmentation[C] //Proceedings of the IEEE Conference on Computer Vision and Pattern Recognition. Los Alamitos: IEEE Computer Society Press, 2019: 3146-3154

[27] Sirinukunwattana K, Pluim J P W, Chen H, et al. Gland segmentation in colon histology images: the glas challenge contest[J]. Medical Image Analysis, 2017, 35: 489-502

[28] Badrinarayanan V, Kendall A, Cipolla R. SegNet: a deep convolutional encoder-decoder architecture for image segmentation[J]. IEEE Transactions on Pattern Analysis and Machine Intelligence, 2017, 39(12): 2481-2495 